\title{
Blood refractive index modelling in the visible and near infrared spectral regions
}

\author{
Ekaterina N. Lazareva ${ }^{1,2,3 *}$, and Valery V. Tuchin ${ }^{1,4,5}$ \\ ${ }^{1}$ Research Educational Institute of Optics and Biophotonics, Saratov State University (National Research University), \\ 83 Astrakhanskaya Str., Saratov 410012, Russia \\ ${ }^{2}$ Center for Functionalized Magnetic Materials (FunMagMa), Immanuel Kant Baltic Federal University, \\ 14 A. Nevskogo Str., Kaliningrad 236041, Russia \\ ${ }^{3}$ Interdisciplinary Laboratory of Biophotonics, Tomsk State University (National Research University), 36 Lenin av., \\ Tomsk 634050, Russia \\ ${ }^{4}$ Laboratory of Laser Diagnostics of Technical and Living Systems, Institute of Precision Mechanics and Control, \\ Russian Academy of Sciences, 24 Rabochaya Str., Saratov 410028, Russia \\ ${ }^{5}$ Samara National Research University, 34 Moskovskoye Shosse, Samara 443086, Russia
}

* e-mail: LazarevaEN@list.ru

\begin{abstract}
We present the calculation of blood refractive index based on the experimental data for the real part of the refractive index of the main protein components, haemoglobin and albumin. The refractive index of blood proteins was measured using the multi-wavelength Abbe refractometer at the wavelengths 480, 486, 546, 589, 644, $656,680,930,1100,1300$, and $1550 \mathrm{~nm}$ at room temperature $+24^{\circ} \mathrm{C}$. (C) 2018 Journal of Biomedical Photonics \& Engineering.
\end{abstract}

Keywords: refractive index; blood; blood components; blood dispersion; visible/NIR wavelengths; Sellmeier formula.

Paper \#3278 received 15 Jan 2018; revised manuscript received 14 Mar 2018; accepted for publication 16 Mar 2018; published online 26 Mar 2018. doi: 10.18287/JBPE18.04.010503. [Special Section. Workshop "Biophotonics" of the XV all-Russian Youth Samara conference-contest on optics and laser physics].

\section{References}

1. V. V. Tuchin, Tissue Optics: Light Scattering Methods and Instruments for Medical Diagnostics, $3^{\text {rd }}$ Edition, SPIE Press, Bellingham, WA (2015).

2. J. Heijmans, L. Cheng, and F. Wieringa, "Optical fiber sensors for medical applications - Practical engineering considerations," IFMBE Proceedings 22, 2330-2334 (2009).

3. V. V. Tuchin (Ed.), Handbook of Optical Biomedical Diagnostics. Light-Tissue Interaction, vol. 1, 2nd Edition, SPIE Press, Bellingham, WA (2016).

4. A. N. Yaroslavsky, I. V. Yaroslavsky, T. Goldbach, and H.-J. Schwarzmaier, "Optical properties of blood in the near infrared spectral range," Proceedings of SPIE 2678, 314-324 (1996).

5. S. L. Jacques, "Corrigendum: Optical properties of biological tissues: a review," Physics in Medicine and Biology 58(13), 5007-5008 (2013).

6. N. Bosschaart, G. J. Edelman, M. C. G. Aalders, T. G. van Leeuwen, and D. J. Faber, "A literature review and novel theoretical approach on the optical properties of whole blood," Lasers in Medical Science 29(2), 453479 (2014).

7. J. Wang, Zh. Deng, X. Wang, Q. Ye, W. Zhou, J. Mei, Ch. Zhang, and J. Tian, "Measurement of the refractive index of hemoglobin solutions for a continuous spectral region," Biomedical Optics Express 6(7), 2536-2541 (2015).

8. O. Zhernovaya, O. Sydoruk, V. Tuchin, and A. Douplik, "The refractive index of human hemoglobin in the visible range," Physics in Medicine and Biology 56(13), 4013-4021 (2011).

9. M. Yahya, and M. Z. Saghir, "Empirical modelling to predict the refractive index of human blood," Physics in Medicine and Biology 61(4), 1405-1415 (2016).

10. J. D. Rowe, D. Smith, and J. S. Wilkinson, "Complex refractive index spectra of whole blood and aqueous solutions of anticoagulants, analgesics and buffers in the mid-infrared," Scientific Reports 7, 7356 (2017). 
11. Y. L. Jin, J. Y. Chen, L. Xu, and P. N. Wang, "Refractive index measurement for biomaterial samples by total internal reflection," Physics in Medicine and Biology 51(20), 371-379 (2006).

12. D. J. Faber, M. C. G. Aalders, E. G. Mik, B. A. Hooper, M. J. C. van Gemert, and T. G. van Leeuwen, "Oxygen saturation-dependent absorption and scattering of blood," Physical Review Letters 93, 028102 (2004).

13. M. Jedrzejewska-Szczerska, "Measurement of complex refractive index of human blood by low-coherence interferometry," The European Physical Journal Special Topics 222(9), 2367-2372 (2013)

14. Zh. Wang, K.Tangella, A. Balla, and G. Popescu, "Tissue refractive index as marker of disease," Journal of Biomedical Optics 16(11), 116017 (2011).

15. H. Majeed, Sh. Sridharan, M. Mir, L. Ma, E. Min, W. Jung, and G. Popescu, "Quantitative phase imaging for medical diagnosis," Journal of Biophotonics 10(2), 177-205 (2017).

16. G. Mazarevica, T. Freivalds, and A. Jurka, "Properties of erythrocyte light refraction in diabetic patients," Journal of Biomedical Optics 7(2), 244-247 (2002).

17. L. V. Plotnikova, A. M. Polyanichko, M. O. Kobeleva, A. A. Nikekhin, M. V. Uspenskaya, A. V. Kayava, A. D. Garifullin, and S. W. Voloshin, "Analysis of blood serum by the method of refractometry in antitumor therapy in patients with multiple myeloma," Optics and Spectroscopy 124(1), 140-142 (2018).

18. A. N. Bashkatov, E. A. Genina, and V. V. Tuchin, "Optical properties of skin, subcutaneous, and muscle tissues: a review," Journal of Innovative Optical Health Sciences 4(1), 9-38 (2011).

19. F. P. Bolin, L. E. Preuss, R. C. Taylor, and R. J. Ference, "Refractive index of some mammalian tissues using a fiber optic cladding method," Applied Optics 28(12), 2297-2303 (1989).

20. D. K. Sardar, and L. B. Levy, "Optical properties of whole blood," Lasers in Medical Science 13(2), 106-111 (1998).

21. H. Li, L. Lin, and S. Xie, "Refractive index of human whole blood with different types in the visible and nearinfrared ranges," Proceedings of SPIE 3914, 517-521 (2000).

22. S. Cheng, H. Y. Shen, G. Zhang, C. H. Huang, and X. J. Huang, "Measurement of the refractive index of biotissue at four laser wavelengths," Proceedings of SPIE 4916, 172-176 (2002).

23. R. Barer, "Refractometry and interferometry of living cells," Journal of the Optical Society of America 47(6), 545-556 (1957).

24. M. V. Volkenshtein, Molecular Optics, Gosteskhizdat, Moscow (1951) [in Russian].

25. D. Segelstein, "The complex refractive index of water," M.S. thesis, Department Of Physics, University of Missouri, Kansas City (1981).

26. N. G. Khlebtsov, I. L. Maksimova, I. V. Meglinski, L. Wang, and V. V. Tuchin, "Introduction to light scattering by biological objects," Chapter 1 in Handbook of Optical Biomedical Diagnostics. Light-Tissue Interaction, 2nd Edition, SPIE Press, Bellingham, WA (2016).

27. W. Heller, "Remarks on refractive index mixture rules," The Journal of Physical Chemistry 69(4), 1123-1129 (1966).

28. G. J. Tearney, M. E. Brezinski, J. F. Southern, B. E. Bouma, M. R. Hee, and J. G. Fujimoto, "Determination of the refractive index of highly scattering human tissue by optical coherence tomography," Optics Letters 20(21), 2258-2260 (1995).

29. H.-C. Cheng, and Y.-C. Liu, "Simultaneous measurement of group refractive index and thickness of optical samples using optical coherence tomography," Applied Optics 49, 790-797 (2010).

30. I. Yu. Yanina, N. A. Trunina, and V. V. Tuchin, "Photoinduced cell morphology alterations quantified within adipose tissues by spectral optical coherence tomography," Journal of Biomedical Optics 18(11), 111407 (2013).

31. J. H. Jung, K. Kim, J. Yoon, and Y. K. Park, "Hyperspectral optical diffraction tomography," Optics Express 24(3), 2006-2012 (2016).

32. Y. K. Park, T. Yamauchi, W. Choi, R. Dasari, and M. S. Feld, "Spectroscopic phase microscopy for quantifying hemoglobin concentrations in intact red blood cells," Optics Letters 34(23), 3668-3670 (2009).

33. F. E. Robles, C. Wilson, G. Grant, and A. Wax, "Molecular imaging true-colour spectroscopic optical coherence tomography," Nature Photonics 5(12), 744-747 (2011).

34. F. E. Robles, L. L. Satterwhite, and A. Wax, "Non-linear phase dispersion spectroscopy," Optics Letters 36(23), 4665-4667 (2011).

35. J. Singh, Optical Properties of Condensed Matter and Applications, Wiley, Chichester, (2006).

36. J. A. Lamasso, "Error in hematocrit value produced by excessive ethylenediaminetetraacetate," American Journal of Clinical Pathology 44, 109-110 (1965). 


\section{Introduction}

Optical properties of blood can be considered in a microscopic or macroscopic way. As a microscopic object, blood is a strongly scattering medium. It is a heterogeneous medium, consisting of plasma and blood corpuscles. The blood plasma consists of $90 \%$ water and $10 \%$ proteins. The blood corpuscles are mainly red blood cells (almost 99\%), leucocytes (1\%) and thrombocytes $(>1 \%)$. The red blood cells possess the largest geometric size, as a rule, 6.2-8.2 $\mu \mathrm{m}$, and mainly determine the optical properties of blood [1-3]. The quantitative and qualitative information on the blood optical properties, in particular, on the refractive index, is of great interest for many fields of biomedical studies and practical medicine, since the noninvasive or lowinvasive optical technologies become wider and wider used in diagnostics and therapy [1-6]. It is well known that the optical properties of blood are determined by such physiologic and biologic parameters as haematocrit, temperature, osmolarity, saturation with oxygen or other gases, membrane rigidity of the red blood cells, and depend on the wavelength in a complex way [1, 4-12]. The visible and near infrared spectral regions are often referred to as "therapeutic/diagnostic window", since just in this wavelength range water, which is a major component of many biological tissues, absorbs weakly. At present there is a revival of interest to the problem of measuring the refractive index of different biological tissues and blood in a wide range of wavelengths, since just the refractive index is proposed for using as an endogenous diagnostic marker of different diseases [13-17]. For example, in Ref. [17] it is shown that the refractive index of blood serum can be used as additional criterion to assess the dynamics of the variation of blood serum properties under the antitumour therapy. The knowledge of the refractive index of blood in a wide range of wavelengths (optical dispersion) is necessary to describe the optical properties of different layers of blood-saturated tissues, e.g., using the Monte Carlo method of statistical modelling [1, 18]. The knowledge of tissue and blood optical properties allows for determination of the optimal wavelength providing the maximal penetration depth of laser radiation. This is important for modelling the interaction between the tissue and laser radiation, e.g., in planning such clinical procedures as laser interstitial heating or photodynamic therapy, as well as in choosing the operating wavelengths of pulse oximeters widely used in different fields of medicine for monitoring the blood oxygen saturation [1-6].

Thus, the determination of optical dispersion of blood and its components in the visible and near infrared spectral regions is an urgent problem, since there are no complete data in the literature. Moreover, the known data on blood refractive index strongly differ among themselves [8-10,19-22], which additionally motivates the studies.

The refractive index is a complex quantity $\tilde{n}=n+i k$; its real and imaginary part can be described within the frameworks of electron theory as [23-25]:

$$
\begin{aligned}
& n=1+\frac{2 \pi q^{2} N\left(\omega_{0}^{2}-\omega^{2}\right)}{m\left(\omega_{0}^{2}-\omega^{2}\right)^{2}+\gamma^{2} \omega^{2}}, \\
& k=\frac{2 \pi q^{2} N \gamma \omega}{m\left(\omega_{0}^{2}-\omega^{2}\right)^{2}+\gamma^{2} \omega^{2}},
\end{aligned}
$$

where $q$ is the molecule charge; $N$ is the number of molecules per unit volume; $\omega_{0}$ is the natural vibration frequency of the oscillator; $\omega$ is the frequency of the external field oscillation; $m$ is the molecular mass; $\gamma$ is the damping coefficient; $n$ is the real part of refractive index characterising the wave phase shift; $k$ is the imaginary part of refractive index, characterising the wave absorption.

The refractive index of a multicomponent biological medium can be calculated using the Gladstone-Dale law, according to which in the absence of chemical interaction between the components of the medium the resulting refractive index is the average of refractive indices of the components with their volume fractions as weighting factors, i.e., [26,27]

$$
n=\sum_{i=1}^{N} n_{i} f_{i}
$$

where $n_{i}$ and $f_{i}$ are the refractive indices and the volume fractions of individual components, respectively, and $N$ is the number of components.

In a simplified model, the blood can be presented as a two-component medium consisting of red blood cells suspended in plasma. Then Eq. (3) can be applied to the blood in the form

$$
n_{\text {blood }}=n_{R B C} f_{R B C}+n_{P l} f_{P l}
$$

where $n_{\text {blood }}, n_{R B C}$, and $n_{P l}$ are the refractive indices of blood, red blood cells, and plasma, respectively; $f_{R B C}$ and $f_{P l}$ are the volume fractions of red blood cells and plasma in the blood, respectively.

Since the plasma consists of $90 \%$ water and $10 \%$ proteins, the main of which is albumin, its refractive index can be calculated using the Gladstone-Dale formula written in the form:

$$
n_{P l}=0.9 n_{\text {Water }}+0.1 n_{A l b}
$$

where $n_{P l}, n_{\text {Water }}$, and $n_{A l b}$ are the refractive indices of plasma, water and pure albumin, respectively.

The refractive index of red blood cells can be also calculated using Eq. (3) keeping in mind that haemoglobin, the main protein in the red blood cell, occupies $25 \%$ of its volume, and the rest volume is filled with water [1-3]:

$$
n_{R B C}=0.75 n_{\text {Water }}+0.25 n_{H b}
$$


where $n_{R B C}, n_{\text {Water }}$, and $n_{H b}$ are the refractive indices of the red blood cell, water, and pure haemoglobin, respectively.

Since the blood is a biological extremely turbid medium with high anisotropy of scattering $(g=0.9996)$ [3], possessing strong scattering and absorption in the visible region, different models of light scattering by particles and other indirect methods are used to determine the refractive index and its dispersion dependence. They include, e.g., the empirical methods based on the calculation of blood refractive index from the experimental values of the refractive indices of the components [10] or the theoretical methods that allow for calculation of the real part of the refractive index from the measured spectra of its imaginary part (absorption spectra) using the Kramers-Kronig relations $[10,11,12]$.

Among the direct methods, the most frequently used ones are different modifications of the optical coherence tomography (OCT) [1, 13, 28-33] and phase microscopy $[15,34,35]$, the laser refractometer with hollow prism $[20,21]$, and the methods exploiting the principle of total internal reflection $[8,11,22]$. These methods possess a number of advantages, but are not free of drawbacks. The OCT-based methods allow for refractive index measurements in scattering media, but with the accuracy restricted to $0.01-0.001$, which is not always sufficient in analytical applications. However, in the in vivo measurements the OCT offers wide possibilities for medical applications, where the above accuracy of the refractive index measurement is sufficient [1, 13, 28-33]. Various modifications of phase microscopy are most frequently used to study the refraction properties of individual cells of blood, e.g., the red blood cells $[15,34,35]$. The application of the laser refractometer with hollow prism allows for refractive index measurement in fluids having large coefficients of scattering and absorption, to which the blood belongs. The method provides in vitro measurements with the accuracy to 0.01-0.0001. The drawbacks of the method include the necessity to use laser sources of radiation with relatively high power $[20,21]$. The methods based on total internal reflection allow for in vitro measurement of the refractive index of blood and its individual components with the accuracy 0.0001-0.00001. The simplicity and the small amount of the required sample material make them available for on-line monitoring of the refractive index [8, 11, 22].

In the present paper, we report the experimental studies of the real part of the refractive index of haemoglobin and albumin, the main protein components of blood. Basing on these data, we calculate the refractive index of whole blood. The refractive index of blood proteins was measured using the multiwavelength Abbe refractometer for 11 wavelengths in the visible and near infrared ranges at room temperature $+24^{\circ} \mathrm{C}$.

\section{Methods and Materials}

For the experimental studies, the haemoglobin solution was prepared from whole blood of a healthy human. The whole blood was collected in test tubes containing heparin to prevent coagulation. The blood sample was centrifuged during $10 \mathrm{~min}$ at $2000 \mathrm{rpm}$ to separate the blood into fractions and to separate the red blood cells from plasma and other blood corpuscles. The red blood cells were gathered in a separate test tube, and then their haemolysis was implemented by multiple freezing and defrosting of erythrocyte mass at the temperature $-18^{\circ} \mathrm{C}$. The haemoglobin concentration in the studied samples was calculated using the spectral method from the absorption spectra and amounted to $260 \mathrm{~g} / \mathrm{l}$, which corresponds to the mean haemoglobin concentration in a red blood cell.

To prepare the albumin solution we used the dry human serum albumin (Sigma-Aldrich), dissolved in $0.9 \% \mathrm{NaCl}$ saline to the concentration of $55 \mathrm{~g} / \mathrm{l}$. The obtained concentration corresponded to the mean value of the albumin concentration in the blood plasma.

The refractive index was measured using the multiwavelength Abbe refractometer (Atago, Japan). In the setup, the source of radiation was a high-power candescent lamp. To select the wavelength we used narrow-band interference filters for 480, 486, 546, 589, $644,656,680,930,1100,1300$, and $1500 \mathrm{~nm}$. The measurement error introduced by the instrument amounts to \pm 0.0002 .

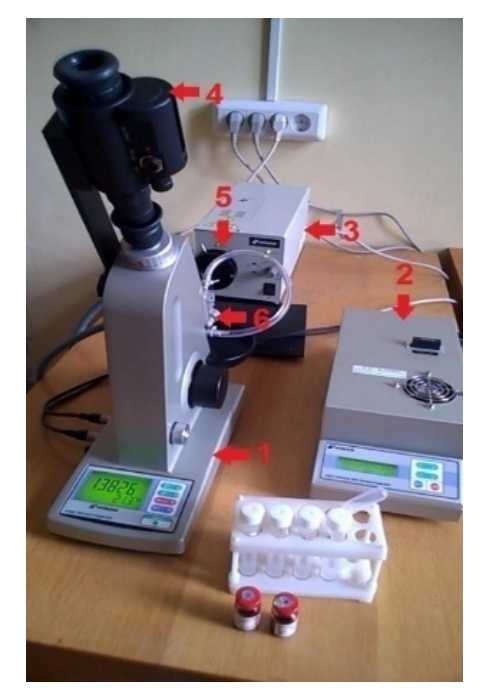

Fig. 1 General view of the experimental setup: 1 multi-wavelength Abbe refractometer; 2 - power supply; 3 - light source; 4 - special head for measurements in the near IR range; 5 - interference filter; 6-sample.

In the beginning of every measurement, the calibration of the instrument using the known tabulated value of the refractive index of distilled water was executed. The sample temperature during the measurements was kept to be $+24^{\circ} \mathrm{C}$ by means of water 
circulation in the refractometer provided by a thermostat.

The blood refractive index was calculated using Eqs. (4), (5), and (6). The volume fractions of plasma and red blood cells were taken to be 0.55 and 0.45 , which corresponds to the haematocrit $45 \%$. The refractive index of pure albumin was calculated using the Gladstone-Dale formula from the measured refractive index of the albumin solution with the concentration $55 \mathrm{~g} / 1$ :

$$
n_{A l b}=\left(n_{\text {Alb sol }}-f_{\text {Water }} n_{\text {Water }}\right) / f_{A l b},
$$

where $n_{A l b}, n_{A l b \text { sol }}, n_{\text {Water }}$ are the refractive indices of pure albumin, albumin solution and water, respectively; $f_{\text {Water }}$, $f_{A l b}$ are the volume fractions of water and pure albumin in the plasma, respectively.

The refractive index of pure haemoglobin for Eq. (6) was calculated from the measured refractive index of the haemoglobin solution with the concentration $260 \mathrm{~g} / \mathrm{l}$ :

$$
n_{H b}=\left(n_{H b \text { sol }}-f_{\text {Water }} n_{W a t e r}\right) / f_{H b}
$$

where $n_{H b}, n_{H b s o l}, n_{\text {Water }}$ are the refractive indices of haemoglobin in a red blood cell, haemoglobin solution, and water, respectively; $f_{W a t e r}, f_{H b}$ are the volume fractions of water and haemoglobin in a red blood cell.

The refractive index of blood was approximated by the Sellmeier formula:

$$
n^{2}(\lambda)=1+\frac{A 1 * \lambda^{2}}{\lambda^{2}-B 1}+\frac{A 2 * \lambda^{2}}{\lambda^{2}-B 2}
$$

where $A 1, A 2, B 1, B 2$ are empirical constants. The Sellmeier formula yields the best result for the description of the dispersion curve of a multicomponent system near the absorption band of each component [35].

\section{Results and Discussion}

The values of refractive index for the haemoglobin and albumin solutions measured at 11 wavelengths of the visible and near infrared range are presented in Table 1. The values of the blood refractive index also presented in Table 1 were calculated using Eq. (4).

Fig. 2 displays the dispersion dependence of the blood refractive index for the calculated values of the refractive index (squares) and the best approximation (red solid line), implemented using the Sellmeier formula (9). As seen from the plot, for the visible and near infrared regions the dispersion of the blood refractive index demonstrates nonlinear behaviour, and the refractive index value decreases with the growth of the wavelength.

After determination of the empirical constants, the Sellmeier Eq. (9) takes the form

$$
\begin{aligned}
n^{2}(\lambda) & =1+\frac{0.83423 * \lambda^{2}}{\lambda^{2}-10775.44775}+ \\
& +\frac{0.04296 * \lambda^{2}}{\lambda^{2}-6.13587 * 10^{6}}
\end{aligned}
$$

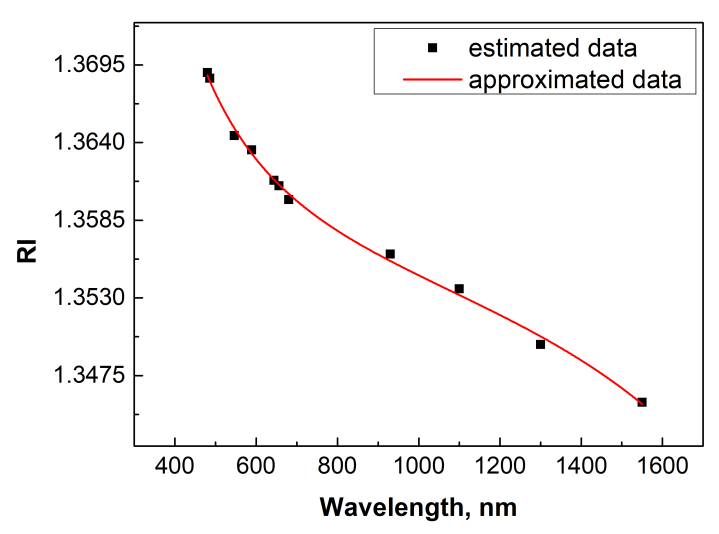

Fig. 2 Dispersion curve of blood refractive index.

The correlation coefficient for fitting of experimental data to the Sellmeier formula equals $\mathrm{R}^{2}=0.99$.

Fig. 3 presents the comparison of the refractive index obtained in the present paper with those found in the literature [9, 19-22].

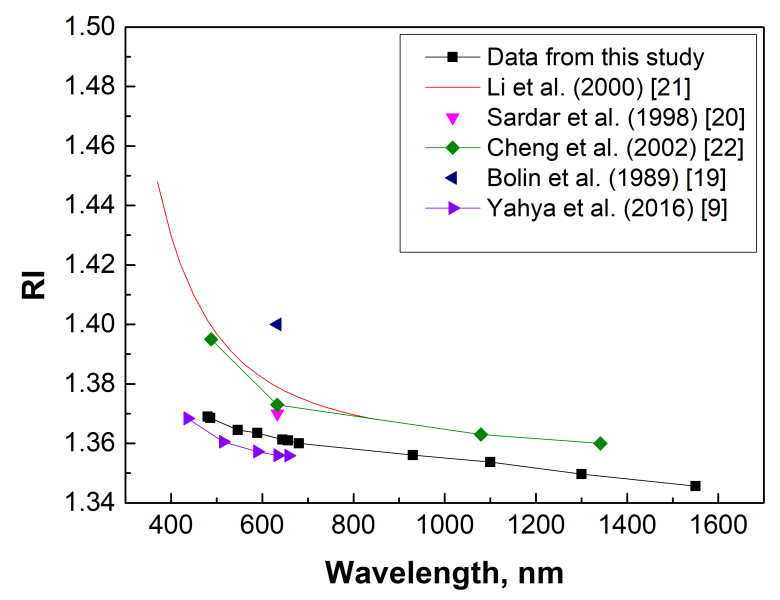

Fig. 3 Dispersion curve of blood in comparison with the data from literature

As already mentioned above, the measurement of blood refractive index is a complex problem solved using complementary direct and indirect methods. The advantage of direct methods is the measurement of the refractive index in the presence of all blood components. However, the inhomogeneity of the cell suspension medium, the strong absorption and scattering do not allow for measurement of whole blood using a direct method with high accuracy, so that the values of the refractive index can strongly differ depending on the sample condition and measurement 
Table 1 Refractive indices of haemoglobin and albumin solutions measured at temperature $+24^{\circ} \mathrm{C}$ and of blood calculated using these measurements.

\begin{tabular}{cccc}
\hline $\begin{array}{c}\text { Wavelength, } \\
\mathbf{n m}\end{array}$ & $\begin{array}{c}\text { Refractive index of haemoglobin } \\
\text { solution in water }(\mathbf{2 6 0} \mathbf{g} / \mathbf{l})\end{array}$ & $\begin{array}{c}\text { Refractive index of albumin } \\
\text { solution in water } \mathbf{( 5 5} \mathbf{g} / \mathbf{l})\end{array}$ & $\begin{array}{c}\text { Refractive index of } \\
\text { blood }\end{array}$ \\
\hline 480 & $1.3945( \pm 0.0002)$ & $1.3480( \pm 0.0002)$ & $1.3690( \pm 0.0002)$ \\
\hline 486 & $1.3939( \pm 0.0002)$ & $1.3478( \pm 0.0002)$ & $1.3686( \pm 0.0002)$ \\
\hline 546 & $1.3885( \pm 0.0002)$ & $1.3449( \pm 0.0002)$ & $1.3645( \pm 0.0002)$ \\
\hline 589 & $1.3880( \pm 0.0003)$ & $1.3434( \pm 0.0002)$ & $1.3635( \pm 0.0002)$ \\
\hline 644 & $1.3854( \pm 0.0003)$ & $1.3416( \pm 0.0002)$ & $1.3613( \pm 0.0002)$ \\
\hline 656 & $1.3849( \pm 0.0004)$ & $1.3414( \pm 0.0002)$ & $1.3610( \pm 0.0002)$ \\
\hline 680 & $1.3837( \pm 0.0002)$ & $1.3405( \pm 0.0002)$ & $1.3600( \pm 0.0002)$ \\
\hline 930 & $1.3782( \pm 0.0005)$ & $1.3380( \pm 0.0004)$ & $1.3561( \pm 0.0003)$ \\
\hline 1100 & $1.3751( \pm 0.0006)$ & $1.3361( \pm 0.0003)$ & $1.3537( \pm 0.0003)$ \\
\hline 1300 & $1.3707( \pm 0.0004)$ & $1.3325( \pm 0.0004)$ & $1.3497( \pm 0.0003)$ \\
\hline 1550 & $1.3665( \pm 0.0005)$ & $1.3285( \pm 0.0004)$ & $1.3456( \pm 0.0003)$ \\
\hline
\end{tabular}

technique. The results of direct measurement of blood refractive index are also affected by two additional important phenomena, the aggregation and sedimentation. The necessary usage of anticoagulants can lead to the deformation of some blood corpuscles, which affects the optical properties. For example, it is known that the use of heparin can change the size and shape of thrombocytes and leucocytes, and the excess amount of $\mathrm{K}_{2}$ EDTA can be hypertonic and lead to osmotic stress. The sedimentation is related to the fact that the density of blood cells is higher than the density of plasma and saline. Although the sedimentation rate in normal blood is not high (up to $30 \mathrm{~mm} / \mathrm{h}$ ), it can be essential in the long-time measurements of the sample optical properties $[3,36]$. Bolin et al. obtained the value of blood refractive index equal to 1.400 using a fibreoptical laser refractometer at the wavelength $632.8 \mathrm{~nm}$ [19]. Sardar et al. measured the refractive index of a few concentrations of the diluted solutions of whole blood by means of the laser refractometer with hollow prism. Thus, for the $60 \%$ blood solution they found the refractive index 1.37 at the wavelength $632.8 \mathrm{~nm}$ [20]. Li et al. performed the refractive index measurement in the samples of different groups of blood (O(I), A(II), and $\mathrm{B}(\mathrm{III})$ ) using the method based on the principle of total internal reflection; they presented a dispersion formula for calculating the averaged refractive index of blood in the visible and near infrared spectral region. According to this formula, they determined the value of refractive index 1.4480-1.3680 for the wavelength range 370-850 nm [21]. Cheng et al. measured the refractive index of the whole blood using the method of total internal reflection with several lasers. They obtained the following values of the refractive index: 1.395 for 488 $\mathrm{nm}, 1.373$ for $632.8 \mathrm{~nm}, 1.363$ for $1079.5 \mathrm{~nm}, 1.360$ for $1341.4 \mathrm{~nm}[22]$.

The modelling methods allow for reconstruction of blood refractive index in a wide range of wavelengths without considering the medium inhomogeneity from the known data for each of its components. In the present paper, using the modelling method with the values of albumin and haemoglobin refractive index measured by means of refractometry, we obtained the values of blood refractive index 1.3690-1.3456 for the wavelength range $480-1550 \mathrm{~nm}$. Recently Yahya et al. presented the calculation of the real part of refractive index at different haemoglobin concentrations, temperatures, and wavelengths basing on the experimental values of refractive index of dry haemoglobin aqueous solutions, measured by means of the Abbemat refractometer. For example, at room temperature, for the haemoglobin concentration of 150 $\mathrm{g} / 1$, and the wavelengths $436.1,513.9,589.1,633.2$, and $657.2 \mathrm{~nm}$ they found the values of blood refractive index $1.36481,1.36053,1.35724,1.35601$, and 1.35587, respectively [9]. The values of the refractive index obtained in the present paper do not strongly differ from those of Ref. [9], which can be seen in Fig. 3. The discrepancies can be due to the difference in the preparation protocols of blood samples and blood components, the specific features of experimental setups and modelling methods.

The results of the study show that the proposed method of modelling the refractive index in 
multicomponent media is suitable for the calculation of blood refractive index in the visible and near infrared spectral regions and can be used for express estimates of blood refractive index. The obtained data complete well the ones already reported in the literature and are important for the calculations of light distribution in blood-saturated tissues, interpretation of results of optical coherence tomography, fluorescence diagnostics, diaphanoscopy in the near infrared spectral region, as well as for providing precision dosimetry in photodynamic therapy and laser thermotherapy.

\section{Conclusion}

The present study demonstrates the possibility of blood refractive index modelling based on the measured values of refractive index of albumin and haemoglobin aqueous solutions. For the dispersion dependence of blood refractive index, we obtained the coefficients that allow for data extrapolation by the Sellmeier formula in the visible and near infrared wavelength range. The results agree well with the known literature data and can be used in further study of dispersion in biological media.

\section{Disclosures}

The authors have no relevant financial interests in this article and no potential conflicts of interest to disclose.

\section{Acknowledgments}

The authors are grateful for the support of this work within the 5 top 100 Russian Academic Excellence Project at the Immanuel Kant Baltic Federal University (ENL), and the Research Programme of the Institute of Precision Mechanics and Control of RAS (VVT). 